\title{
Threshold-Based Registration (TBR) in Mobile IPv6
}

\author{
Linfeng Yang, Jouni Karvo, Teemu Tynjälä, and Hannu Kari \\ Helsinki University of Technology, Telecommunications Software and Multimedia \\ Laboratory, P.O.Box 9700, 02015 HUT, Finland \\ \{lyang, kex, tjtynjal, hhk\}@tcm.hut.fi \\ http://www.tcm.hut.fi/english.html
}

\begin{abstract}
The underlying principles of IETF Internet Draft, Mobility Support in IPv6, make it possible to employ some mechanisms to improve handoff smoothness, to maintain optimized data transfer route, at the mean time without requiring any special support from the network side. Such mechanisms were introduced and examined in this paper together with the proposal of Threshold-Based Registration in Mobile IPv6. In this proposal, a mobile node will establish immediate forwarding from the previous care-of address whenever it moves to another subnetwork. After every fixed number of immediate forwarding steps, the mobile node will establish direct forwarding from its primary care-of address. Again, after every fixed number of direct forwarding steps, the mobile node will register a new primary care-of address to its home agent. With such an approach, the above mentioned goals are achieved.
\end{abstract}

\section{Introduction}

Internet Protocol version 6 (IPv6) [1, is currently under development. As an evolution of current Internet Protocol ( $\operatorname{IPv} 4)$, it provides many advantages, the most important one being the support of mobility. Internet draft Mobility Support in IPv6 2], also known as MobileIPv6, proposed by the Internet Engineering Task Force (IETF), gives strong support for route optimization compared with its counterpart in Mobile IPv4[3].

In MobileIPv6, not only can the routes between mobile nodes and correspondent nodes be optimized, but also the route between a mobile node and its home agent. As a disadvantage of this optimized route, high signaling load is incurred. A proposal using a hierarchical approach to reduce this kind of signaling load has been introduced 4]. However, this approach has been criticized for contradicting the design goals of MobileIPv6 5 .

The newest version of MobileIPv6 has clarifed how to establish forwarding from the previous care-of address. It intends to reduce packet loss during handoff. We claim that this mechanism can also be employed to reduce the signaling load. This article will present a scheme for using forwarding to reduce signaling load while maintaining optimized data routes between correspondent nodes and mobile nodes. 
The paper is organized as follows: Section 2 describes the current method for mobility support in IPv6. Section 3 presents our threshold based proposal. Section 4 contains a brief discussion on the proposed approach, and Section 5 concludes the paper.

\section{Mobility Support in IPv6}

In this section, we describe the current method for mobility support in MobileIPv6[2]. First, some terminology needs to be defined. A home link is the link by which a mobile node's home subnet prefix is defined. Standard IP routing mechanism will deliver packets destined for a mobile node's home address to its home link. A care-of address means an IP address associated with a mobile node when the mobile node is visiting a foreign link; the subnet prefix of this IP address is a foreign subnet prefix. A router on a mobile node's home link with which the mobile node has registered its current care-of address is called a home agent. The care-of address registered with the mobile node's home agent is called the primary care-of address.

A correspondent node is a node that wants to send a packet or several packets to the mobile node. A binding is the association of the home address of a mobile node with a care-of address for that mobile node, along with the remaining lifetime of that association. Each IPv6 node has a binding cache, which contains binding related information. A binding update is an IPv6 Destination Option used by a mobile node to notify a correspondent node or the mobile node's home agent of its current binding. That is, it can be piggybacked on data packets of the ongoing connection, and only the destination node can process this option. A routing header is an IPv6 option header; it is used by an IPv6 source to list one or more intermediate nodes to be "visited" on the way to a packet's destination[1].

When a correspondent node wants to send packets to a mobile node, it first examines its binding cache for an entry for the destination address to which the packet is being sent. If no entry is found, it simply sends the packets normally, with no routing header. If the mobile node has not registered a care-of address with any home agent, the packets will go to the mobile node's home link as conventional IPv6. If the mobile node is currently at a foreign network and had registered a care-of address to this home agent, the packet will be received by the mobile node's home agent. The home agent then encapsulates the packet and tunnels it to the mobile node's care-of address. Upon receiving the tunneled packet, the mobile node will send a binding update to the correspondent node. The correspondent node can then send the rest of the packets directly to the mobile node using a routing header. See Fig. 1.

MobileIPv6[2] Sec. 10.5, Forming New Care-of Addresses says:

"After detecting that it has moved from one link to another (i.e., its current default router has become unreachable and it has discovered a new default router), a mobile node SHOULD form a new primary care-of address using one of the on-link subnet prefixes advertised by the new 


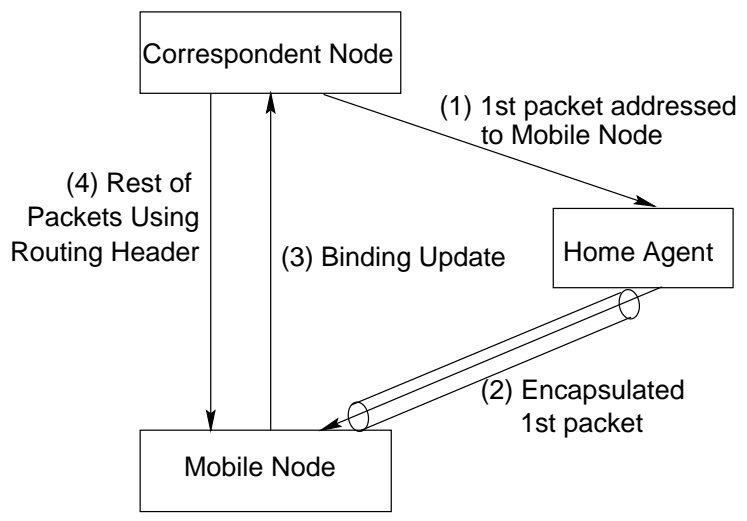

Fig. 1. Routing in MobileIPv6

router. A mobile node MAY form a new primary care-of address at any time, except that it MUST NOT do so too frequently."1

And Sec. 10.6, Sending Binding Updates to the Home Agent says:

"After deciding to change its primary care-of address as described in Sections 10.4 and 10.5, a mobile node MUST register this care-of address with its home agent in order to make this its primary care-of address."

So, if we can form a care-of address and use it to maintain mobile node accessibility, without registering it to the home agent, then the signaling load will be reduced. MobileIPv6 also permits a mobile node to form a new primary care-of address at any time, so we may postpone forming a new primary care-of address when it is possible. Our approach is based on this observation, and will be described in the next section.

\section{Threshold-Based Registration (TBR)}

To maintain mobile node accessibility without forming a new primary care-of address each time a mobile node moves to another subnetwork, we employ the mechanism introduced in MobileIPv6 Section 10.9. Establishing Forwarding from a Previous Care-of Address.

First, we define a new terminology, anchor home agent. When a mobile node moved to a foreign link and registered a primary care-of address to its home agent, the router in this foreign link which can act as the home agent of this mobile node's primary care-of address will be called an anchor home agent. Later,

1 The keywords "SHOULD", "MUST", and "MAY" are frequently used in RFCs. They are required by MobileIPv6 to be interpreted as described in Key words for use in RFCs to indicate requirement levels, RFC $2119[6]$. 
the mobile node can establish direct forwarding from this anchor home agent to its current care-of address. An immediate forwarding means that packets are forwarded from a mobile node's previous care-of address to the current care-of address as described in MobileIPv6 Sec. 10.9.

In the following subsection, we will introduce employment of this forwarding mechanism by giving three approaches. The third and final proposal of TBR is indeed the compromise of the first two.

\subsection{First Approach}

In the first approach a mobile node establishes forwarding only from its immediate previous care-of address. It repeats such forwarding until either there is no router in the previous visited subnet that can act as a home agent or the number of forwarding steps exceeds a predefined limit. This limit is called the maximum number of forwarding steps $F_{\max }$. At this time, the mobile node will form a new primary care-of address using the newest care-of address and sends a binding update to its home agent. See Fig. 2 for a possible scenario.
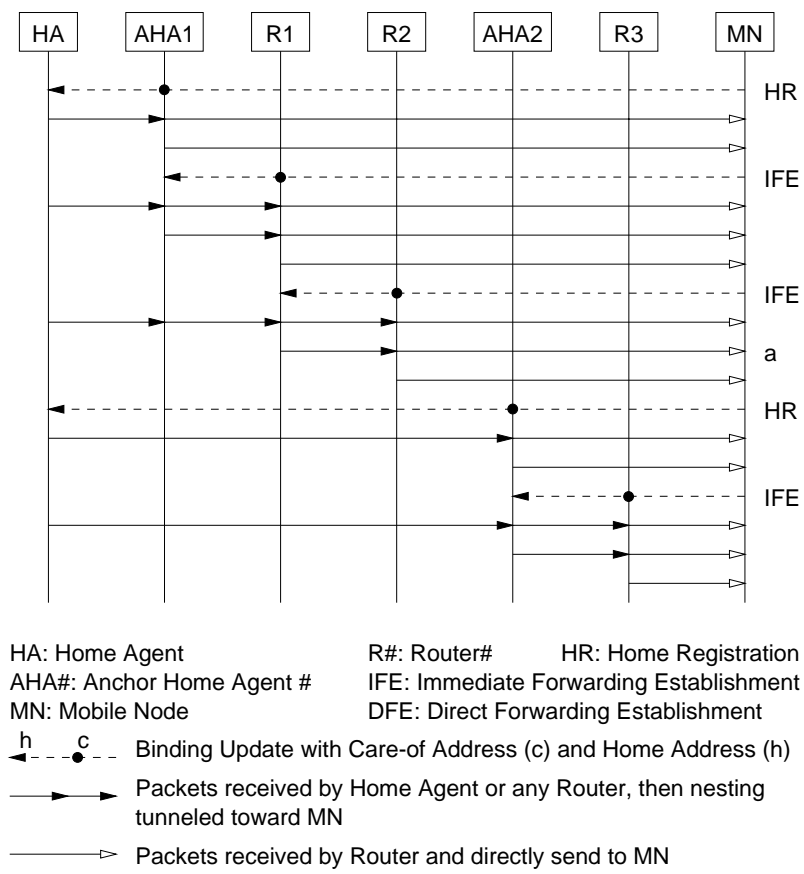

Fig. 2. Establishes forwarding from previous care-of address only. Notice the start point of received packets at $a$, only those close to mobile node's current location are shown in the figure. This is due to direct binding update to the correspondent node, which then sends packets to mobile node's most recent care-of addresses 
In practice, this approach introduced a nested tunnel which may lead to segmentation of data packets and add delay for data processing. Generic Packet Tunneling in IPv6 Specification [7] recommends that the default value of IPv6 Tunnel Encapsulation Limit is 4 . If a packet with Destination Option extension header containing this limit option with value close to 4 is sent by a correspondent node, and the nested tunnel contains more than this number of forwarding, the packets will be discarded before reaching the mobile node. Thus, the benefit of this approach is limited.

\subsection{Second Approach}

Another approach would be that the mobile node establishes forwarding both from the immediate previous care-of address and from its current primary care-of address when it moves to another subnetwork. See Fig. 3 .

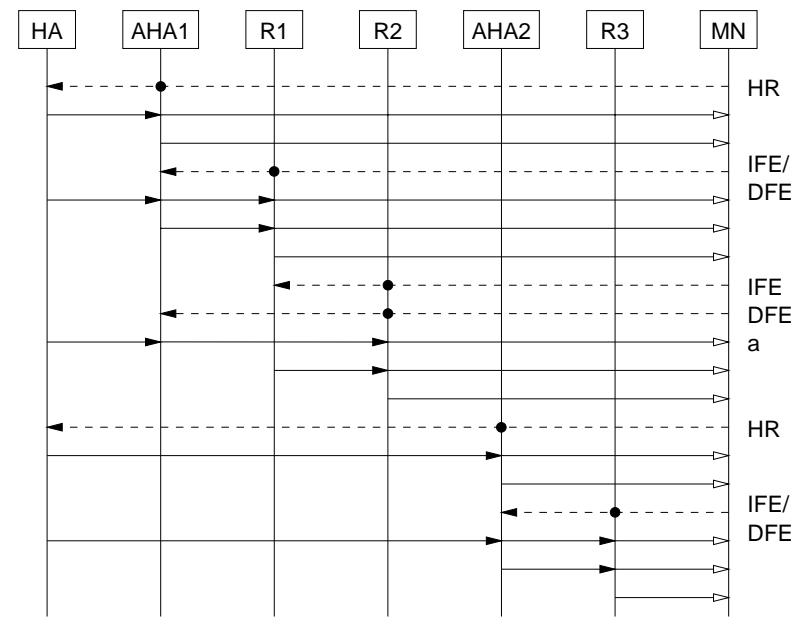

Fig. 3. Establishes forwarding from both immediate previous care-of address and the primary care-of address. At $a$, the packets tunneled from the home agent will need only one extra tunnel to the mobile node, thus solving the problem arising from the first approach. Other symbols are the same as in Fig. 2]

Note that in this approach, the lifetime used in the binding updates for establishing forwarding from immediate previous care-of address can be set short. There is no danger of breaking the chain of forwarding, since there is always a direct route from the anchor home agent to the mobile node. But this approach generates a double signaling load locally, and it also consumes more of the mobile node's resources than the first approach. 


\subsection{TBR - Final Proposal}

Thus, we propose a final approach, the TBR approach. In this approach, a mobile node establishes immediate forwarding from the previous care-of address each time it moves to another subnetwork. After a small number of successful immediate forwarding steps, denoted by $I F_{\max }$, the mobile node will establish a direct forwarding from its current primary care-of address. After a number of successful direct forwarding steps, denoted by $D F_{\max }$, the mobile node will register to its home agent using the latest care-of address as a new primary careof address. The mobile node will register the latest care-of address to its home agent also whenever the remaining lifetime of the last home registration is near

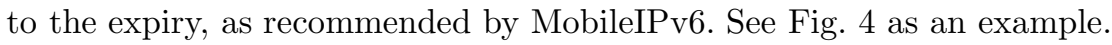

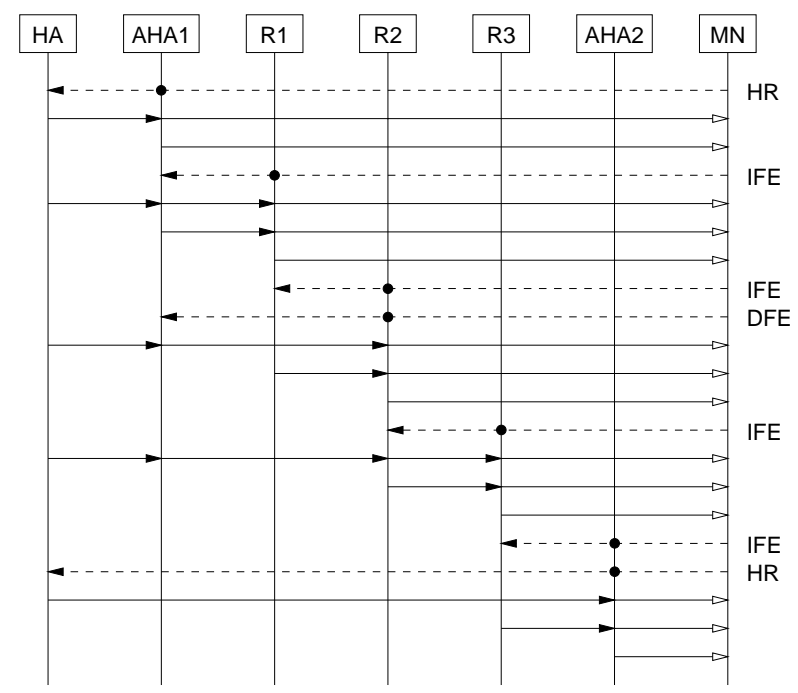

Fig. 4. Mobile node establishes forwarding with TBR approach with $I F_{\max }=2$ and $D F_{\max }=2$. Symbols are the same as in Fig. 2 and Fig. [3]

\subsection{Tunneling Loop Avoidance}

In order to avoid packets tunneled in a loop between two or several routers, a mobile node should act as returning home described in MobileIPv6 Section 10.17 when it switches to a subnetwork from which the forwarding is still valid. It should send a binding update to this router with a zero lifetime, to de-register the previous binding before establishing forwarding from the immediate previous care-of address. 


\section{Discussion}

The benefits of the TBR approach are as follows:

1. Achieving balance between optimized routes and low signaling load.

2. Scalability and reliability. Since a mobile node will change its anchor home agent whenever the lifetime for home registration or the limit number of direct forwarding steps $D F_{\max }$ is reached, the load for the anchor home agents is distributed among the routers in the foreign network. When the number of mobile nodes in the foreign networks increases, there is a smaller probability that the anchor default router will become a bottleneck. When one of the routers is out of order, the mobile node can change its anchor home agent by updating its primary care-of address and sending a new registration to the home agent.

3. Easy implementation. Almost all the information we needed to implement this approach is already stored in the binding update list of the mobile node. Only two counters (number of immediate forwarding and number of direct forwarding) are needed to make a quick decision on which kind of forwarding or registration should be performed for each handoff.

4. Transparent support. Each mobile node can implement this approach without pre-negotiation between the mobile node and the foreign network.

Binding updates used for forwarding establishment should be authenticated in the same way as described for other binding updates in the MobileIPv6. This introduces a need for more security keys to be managed by both the mobile node and the home agent in the foreign network.

Although, we have done some reachability analysis of the approach with formal methods [8], but clearly a thorough performance analysis left for future work, in order to find the optimal values for the parameters $I F_{\max }$ and $D F_{\max }$.

\section{Conclusion}

In this article, we presented the current state-of-the-art MobileIPv6, concentrating on routing and packet forwarding. We presented a modified algorithm for binding updates for reducing signaling load while maintaining data route optimality. The algorithm is, a mobile node establishes immediate forwarding from the previous care-of address whenever it moves to another subnetwork. After every fixed number of immediate forwarding steps, the mobile node establishes direct forwarding from its primary care-of address. Again, after every fixed number of direct forwarding steps, the mobile node registeres a new primary care-of address to its home agent. With such an approach, the above mentioned goals are achieved together with scalabilty, reliability, easy implementation and transparent support as the benefits. 


\section{References}

1. Deering, S., Hinden, R.: Internet Protocol version 6 (IPv6) specification. RFC 2460. 1998

2. Johnson, D., Perkins, C.: Mobility Support in IPv6. Internet Draft. Work in progress. 2000. http://www.ietf.org/internet-drafts/draft-ietf-mobileip-ipv6-10.txt

3. Perkins, C., Editor: IP Mobility Support. RFC 2002. 1996

4. Castelluccia, C., Bellier, L.: Toward a Unified Hierarchical Mobility Management Framework. Internet Draft. Work in progress. 1999. http://www.ietf.org/internetdraft-castelluccia-uhmm-framework-00.txt

5. IETF Online Proceedings, Forty-fifth IETF, Oslo, July 11 - July 16, 1999, Work group 2.5.2 IP Routing for Wireless/Mobile Hosts (mobileip)

6. Bradner, S.: Key words for use in RFCs to indicate requirement levels. RFC 2119. 1997

7. Conta, A., Deering, S.: Generic Packet Tunneling in IPv6 Specification. RFC 2473. 1998. 20

8. Tynjälä, T., Kari, H., Yang, L.: Verification of Threshold-Based Registraion Algorithm in Mobile IPv6. Submitted to IEEE International Conference on Networks' 2000 (September 2000). 2000 